\title{
Kinetic Heavy Quark Mass to Three Loops
}

\author{
Matteo Fael๑, ${ }^{*}$ Kay Schönwald๑, ${ }^{\dagger}$ and Matthias Steinhauser ${ }^{\ddagger}$ \\ Institut für Theoretische Teilchenphysik, Karlsruhe Institute of Technology (KIT), 76128 Karlsruhe, Germany
}

(Received 15 May 2020; revised 24 June 2020; accepted 13 July 2020; published 30 July 2020)

\begin{abstract}
We compute three-loop corrections to the relation between the heavy quark masses defined in the pole and kinetic schemes. Using known relations between the pole and $\overline{\mathrm{MS}}$ quark masses, we can establish precise relations between the kinetic and $\overline{\mathrm{MS}}$ charm and bottom masses. As compared to two loops, the precision is improved by a factor of 2 to 3 . Our results constitute important ingredients for the precise determination of the Cabibbo-Kobayashi-Maskawa matrix element $\left|V_{c b}\right|$ at Belle II.
\end{abstract}

DOI: 10.1103/PhysRevLett.125.052003

Introduction.-Among the main aims of the Belle II experiment at the SuperKEKB accelerator at the High Energy Accelerator Research Organization (Tsukuba) is the precise measurement of various matrix elements in the Cabibbo-Kobayashi-Maskawa (CKM) mixing matrix. These are crucial ingredients for our understanding of $C P$ violation and indispensable input for precision tests of the standard model of particle physics. In this context, the determination of $\left|V_{c b}\right|$, the CKM matrix element entering in $b \rightarrow c$ transitions, at the $1 \%$ level is of particular interest. At present its relative error of about $2 \%$ [1] constitutes an important source of uncertainty in the predictions for $K \rightarrow$ $\pi \nu \bar{\nu}[2,3], B_{s} \rightarrow \mu^{+} \mu^{-}$[4], and $\varepsilon_{K}$ [5], the parameter that quantifies $C P$ violation in kaon mixing. All such processes set strong constraints on new physics with a generic flavor and $C P$ structure.

At present, the values of $\left|V_{c b}\right|$ from inclusive $b \rightarrow c \ell \nu$ decays are obtained from global fits of $\left|V_{c b}\right|$, the bottom and charm masses $\left(m_{b, c}\right)$, and the relevant nonperturbative parameters in the heavy quark expansion. The most recent determination is $\left|V_{c b}\right|=(42.19 \pm 0.78) \times 10^{-3} \quad[1,6-8]$, where the precision is limited by perturbative and power correction uncertainties.

In analyses of $B \rightarrow X_{c} \ell \nu$ decays, it is mandatory to use a so-called "threshold" mass designed such that the perturbative QCD corrections to the decay rate are well-behaved. So far for the analyses, either the kinetic mass $\left(m^{\text {kin }}\right)$ [9] or the $1 S$ mass [10-13] has been chosen. Both schemes are well suited for $B \rightarrow X_{c} \ell \nu$ since they allow for renormalization scales $\mu \leq m_{b}$. The relation between the $1 S$ and $\overline{\mathrm{MS}}$ quark mass $(\bar{m})$ has been computed up to next-to-next-tonext-to-leading order in Refs. [14,15]. For the $m^{\mathrm{kin}}-\bar{m}$

Published by the American Physical Society under the terms of the Creative Commons Attribution 4.0 International license. Further distribution of this work must maintain attribution to the author(s) and the published article's title, journal citation, and DOI. Funded by SCOAP ${ }^{3}$. relation, two-loop corrections and the three-loop terms with two closed massless fermion loops (often referred to as large- $\beta_{0}$ terms) have been computed in Ref. [16].

The rate and the moments of $B \rightarrow X_{c} \ell \nu$ strongly depend on the mass definition of the heavy quark, the choice of which is closely intertwined with the size of the QCD corrections. Perturbative calculations using the on-shell mass scheme are affected by the renormalon ambiguity, which manifests itself through bad behavior of the perturbative series $[17,18]$. However, QCD corrections to the semileptonic rates also exhibit a bad convergence in the $\overline{\mathrm{MS}}$ scheme [9,19]. In fact, large $\left(n \alpha_{s}\right)^{k}$ terms, with $n=5$, arise from the $m^{\mathrm{OS}}-\bar{m}$ conversion of the overall factor $\Gamma \simeq G_{F}^{2} m_{b}^{5}\left|V_{c b}\right|^{2} /\left(192 \pi^{3}\right)$.

The kinetic scheme was introduced in [9] to sum such $n$ enhanced terms via a suitable short-distance definition. It relies on the small velocity QCD sum rules [20], which hold in the zero-recoil limit, i.e., for hadronic final state velocities $|\vec{v}| \ll 1$ in the rest frame of the decaying particle and $m_{b} \sim m_{c}$.

Note that the semileptonic $B$ decays alone precisely determine only a linear combination of the heavy quark masses, approximately given by $m_{b}-0.8 m_{c}$ [6]. Thus, in order to break the degeneracy one must include in the fit external constraints for the bottom and the charm masses, which are usually given in the $\overline{\mathrm{MS}}$ scheme. Until now the scheme-conversion uncertainty from $\bar{m}_{b}\left(\bar{m}_{b}\right)$ to $m_{b}^{\text {kin }}(1 \mathrm{GeV})$ dominates the uncertainty of the $\overline{\mathrm{MS}}$ bottom quark mass [21]. The global fits in [6,7] employed only $\bar{m}_{c}$ as external input as the gain in accuracy with the further inclusion of $\bar{m}_{b}$ would have been limited by scheme conversion [6].

In this Letter we will present the complete three-loop corrections to the $\bar{m}-m^{\text {kin }}$ relation, which lead to a significant improvement of the uncertainties in the mass conversion. Our results constitute a fundamental ingredient for future inclusion of $\mathcal{O}\left(\alpha_{s}^{3}\right)$ corrections in semileptonic rates and spectral moments. Thus, our calculation is one of 
the major steps toward the reduction of the theoretical uncertainties affecting the $\left|V_{c b}\right|$ determination from inclusive decays at the $1 \%$ level or even below.

Kinetic mass definition.-In Ref. [9] (see also Ref. [16]) the kinetic mass has been defined via its relation to the pole mass $m^{\mathrm{OS}}$ through

$$
m^{\mathrm{kin}}(\mu)=m^{\mathrm{OS}}-\left.\bar{\Lambda}(\mu)\right|_{\text {pert }}-\frac{\left.\mu_{\pi}^{2}(\mu)\right|_{\text {pert }}}{2 m^{\text {kin }}}+\cdots,
$$

where the ellipses stand for contributions from higher dimensional operators. The scale $\mu$, the so-called Wilsonian cut-off, is part of the definition of $m^{\mathrm{kin}}$ and takes the role of a normalization point for the kinetic mass. In practice it is of the order of $1 \mathrm{GeV}$.

The quantities $\left.\bar{\Lambda}(\mu)\right|_{\text {pert }}$ and $\left.\mu_{\pi}^{2}(\mu)\right|_{\text {pert }}$ in Eq. (1) correspond to the heavy meson's binding energy and the residual kinetic energy parameters, respectively. They are defined within perturbation theory and are obtained from the following forward scattering amplitude of an external current $J$ and the heavy quark $Q$ [cf. Fig. 1(a)]

$$
T\left(q_{0}, \vec{q}\right)=\frac{i}{2 m} \int \mathrm{d}^{4} x e^{-i q x}\left\langle Q\left|T J(x) J^{\dagger}(0)\right| Q\right\rangle,
$$

where for later convenience we have separated the energy and three momentum components of the external momentum $q$. We furthermore denote the external momentum of the heavy quark by $p$ with $p^{2}=m^{2}$, and we introduce $s=(p+q)^{2}$. We assume that the current $J$ does not change the flavor of the heavy quark with mass $m$. For $\left.\bar{\Lambda}(\mu)\right|_{\text {pert }}$ and $\left.\mu_{\pi}^{2}(\mu)\right|_{\text {pert }}$, one has in the rest frame of the heavy quark $[9,16]$

$$
\begin{aligned}
\left.\bar{\Lambda}(\mu)\right|_{\text {pert }} & =\lim _{\vec{v} \rightarrow 0} \lim _{m \rightarrow \infty} \frac{2}{\vec{v}^{2}} \frac{\int_{0}^{\mu} \omega W(\omega, \vec{v}) \mathrm{d} \omega}{\int_{0}^{\mu} W(\omega, \vec{v}) \mathrm{d} \omega}, \\
\left.\mu_{\pi}^{2}(\mu)\right|_{\text {pert }} & =\lim _{\vec{v} \rightarrow 0} \lim _{m \rightarrow \infty} \frac{3}{\vec{v}^{2}} \frac{\int_{0}^{\mu} \omega^{2} W(\omega, \vec{v}) \mathrm{d} \omega}{\int_{0}^{\mu} W(\omega, \vec{v}) \mathrm{d} \omega},
\end{aligned}
$$

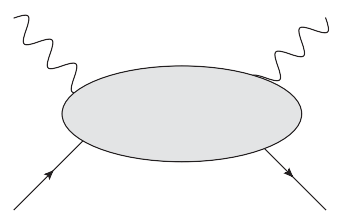

(a)

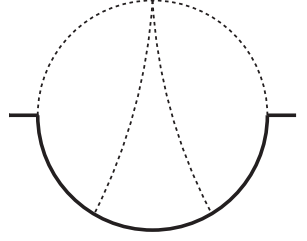

(b)
FIG. 1. (a) Schematic Feynman diagram representing the scattering of an external current, represented by wavy lines and a heavy quark (solid line). The blob represents one-particle irreducible quantum corrections, which we consider up to threeloop order. (b) The most complicated master integral. Dotted lines represent massless relativistic propagators and solid lines stand for eikonal propagators with mass $y$. where the structure function $W$ is given by the discontinuity of $T, W=2 \operatorname{Im}\left[T\left(q_{0}, \vec{q}\right)\right]$. In Eq. (3) we have $\omega=$ $q_{0}-q_{0}^{\min }, \quad \vec{v}=\vec{q} / m, \quad$ and $\quad q_{0}^{\min }=\sqrt{\vec{q}^{2}+m^{2}}-m=$ $m v^{2} / 2+\mathcal{O}\left(v^{4}\right)$. Note that $W$ is zero for $q_{0}<q_{0}^{\min }$.

In order to compute corrections of $\mathcal{O}\left(\alpha_{s}^{3}\right)$ to Eq. (1), one has to consider three-loop corrections to the imaginary part of $T\left(q_{0}, \vec{q}\right)$ in Eq. (2). This requires the evaluation of real and virtual corrections to the scattering process shown schematically in Fig. 1(a).

More details on the derivation of Eq. (3) are provided in Ref. [22].

Calculation.-From Eqs. (1) and (3) we learn that the relation between the kinetic and pole mass is obtained from the imaginary part of the structure function $W(\omega, \vec{v})$ in the limit $\vec{v} \rightarrow 0$. We apply the threshold expansion [23,24], which in our situation reduces to two momentum regions: (i) the loop momenta can be either hard (h) and scale as the quark mass $m$ or (ii) ultrasoft (u) and scale as $y / m$ where $y=m^{2}-s$ measures the distance to the threshold. Note that in our case we have $y<0$. When expanding the denominators, one has to assume that both $p$ and $q$ scale as $m$.

We generate the four-point Feynman amplitudes with qgraf [25] and translate the output to FORM [26] notation. We make sure that the external momenta $p$ and $q$ are routed through the heavy quark line. Afterward, we expand all loop momenta according to the rules of asymptotic expansion, which leads to a decomposition of each integral into regions in which the individual loop momenta either scale as hard or ultrasoft. At one-loop order, there are only two regions. At two loops, we have the regions (uu), (uh), and (hh), and at three loops we have (uuu), (uuh), (uhh), and (hhh). For each diagram, we have cross-checked the scaling of the loop momenta using the program ASY [27]. Note that the contributions where all loop momenta are hard can be discarded since there are no imaginary parts. The mixed regions are expected to cancel after renormalization and decoupling of the heavy quark from the running of the strong coupling constant. Nevertheless, we perform an explicit calculation of the (uh), (uuh), and (uhh) regions and use the cancellation as a cross-check. The physical result for the quark mass relation is solely provided by the purely ultrasoft contributions.

The starting point of our calculation are four-point functions. However, after the various expansions, we obtain two-point functions with external momentum $p$. As a consequence, denominators become linearly dependent and a partial fraction decomposition is needed in order to generate linear independent sets of propagators. They serve as input for FIRE [28] and LITERED [29], which are used for the reduction to master integrals.

After partial fraction decomposition, we end up with 1,2 , and 14 pure ultrasoft integral families at the one-, twoand three-loop orders, respectively. The three-loop families have eight propagators and four irreducible numerators, 
three of which contain scalar products of the loop momenta and the external momentum $q$ and have been introduced to avoid an expensive tensor reduction.

After reduction to master integrals and their subsequent minimization across all families, the amplitude can be expressed in terms of 1, 3, and 20 ultrasoft master integrals at the one-, two-, and three-loop orders, respectively. At one and two loops, all of them can be expressed in terms of $\Gamma$ functions. This is also the case for 11 of the three-loop master integrals. For eight of the remaining integrals, we obtain analytic results for the $\epsilon$ expansion with the help of Mellin-Barnes [24] representations. In these cases, the residues obtained after closing the integration contour can be summed analytically with the packages SIGMA [30] and EVALUATEMULTISUMS [31], together with HARMONICSUMS [32]; additionally, we obtain high-precision numerical results and use the PSLQ [33] algorithm to reconstruct the analytic expression. We have only encountered one integral where a different strategy was necessary. It is shown in graphical form in Fig. 1(b). For this integral we have introduced a different mass scale, $x$, in the bottommiddle propagator. In case this mass is zero $(x=0)$, the integral can be computed analytically. Thus, we construct differential equations [34-36], apply boundary conditions at $x=0$, and evaluate the solution for $x=1$, which provides the desired integral. We will provide more details on the computation of the master integrals in Ref. [22].

Let us mention that we have performed our calculation for a general gauge parameter $\xi$. We expand the amplitude up to linear order in $\xi$ and check that $\xi$ cancels after adding the quark mass counterterms. Furthermore, for the external current $J$ we use both a vector $\left(J=\bar{Q} \gamma_{\mu} Q\right)$ and a scalar $(J=\bar{Q} Q)$ current and check that the final result for the relation between the pole and kinetic mass is the same. However, the intermediate expressions are different. This concerns, e.g., the renormalization of the current itself. Whereas the vector current has a vanishing anomalous dimension, an explicit renormalization constant is needed for the scalar current. Furthermore, in the case of the vector current, there is no contribution from the virtual corrections contained in the denominator of Eq. (3) since in the static limit the Dirac form factor vanishes and the Pauli form factor is suppressed by $q^{2}$. On the other hand, in the scalar case there is a contribution from the finite static form factor.

Results.-The main result of our calculation is the relation between the kinetic and the pole mass, which up to order $\alpha_{s}^{3}$ is given by

$$
\begin{aligned}
\frac{m^{\mathrm{kin}}}{m^{\mathrm{OS}}}= & 1-\frac{\alpha_{s}^{\left(n_{l}\right)}}{\pi} C_{F}\left(\frac{4}{3} \frac{\mu}{m^{\mathrm{OS}}}+\frac{1}{2} \frac{\mu^{2}}{\left(m^{\mathrm{OS}}\right)^{2}}\right)+\left(\frac{\alpha_{s}^{\left(n_{l}\right)}}{\pi}\right)^{2} C_{F}\left\{\frac{\mu}{m^{\mathrm{OS}}}\left[C_{A}\left(-\frac{215}{27}+\frac{2 \pi^{2}}{9}+\frac{22}{9} l_{\mu}\right)+n_{l} T_{F}\left(\frac{64}{27}-\frac{8}{9} l_{\mu}\right)\right]\right. \\
& \left.+\frac{\mu^{2}}{\left(m^{\mathrm{OS}}\right)^{2}}\left[C_{A}\left(-\frac{91}{36}+\frac{\pi^{2}}{12}+\frac{11}{12} l_{\mu}\right)+n_{l} T_{F}\left(\frac{13}{18}-\frac{1}{3} l_{\mu}\right)\right]\right\}+\left(\frac{\alpha_{s}^{\left(n_{l}\right)}}{\pi}\right)^{3} C_{F}\left\{\frac { \mu } { m ^ { \mathrm { OS } } } \left[C _ { A } ^ { 2 } \left(-\frac{130867}{1944}\right.\right.\right. \\
& \left.+\frac{511 \pi^{2}}{162}+\frac{19 \zeta_{3}}{2}-\frac{\pi^{4}}{18}+\left(\frac{2518}{81}-\frac{22 \pi^{2}}{27}\right) l_{\mu}-\frac{121}{27} l_{\mu}^{2}\right)+C_{A} n_{l} T_{F}\left(\frac{19453}{486}-\frac{104 \pi^{2}}{81}-2 \zeta_{3}\right. \\
& \left.\left.+\left(-\frac{1654}{81}+\frac{8 \pi^{2}}{27}\right) l_{\mu}+\frac{88}{27} l_{\mu}^{2}\right)+C_{F} n_{l} T_{F}\left(\frac{11}{4}-\frac{4 \zeta_{3}}{3}-\frac{2}{3} l_{\mu}\right)+n_{l}^{2} T_{F}^{2}\left(-\frac{1292}{243}+\frac{8 \pi^{2}}{81}+\frac{256}{81} l_{\mu}-\frac{16}{27} l_{\mu}^{2}\right)\right] \\
& +\frac{\mu^{2}}{\left(m^{\mathrm{OS}}\right)^{2}}\left[C_{A}^{2}\left(-\frac{96295}{5184}+\frac{445 \pi^{2}}{432}+\frac{57 \zeta_{3}}{16}-\frac{\pi^{4}}{48}+\left(\frac{2155}{216}-\frac{11 \pi^{2}}{36}\right) l_{\mu}-\frac{121}{72} l_{\mu}^{2}\right)+C_{A} n_{l} T_{F}\left(\frac{13699}{1296}-\frac{23 \pi^{2}}{54}\right.\right. \\
& \left.\left.\left.-\frac{3 \zeta_{3}}{4}+\left(-\frac{695}{108}+\frac{\pi^{2}}{9}\right) l_{\mu}+\frac{11}{9} l_{\mu}^{2}\right)+C_{F} n_{l} T_{F}\left(\frac{29}{32}-\frac{\zeta_{3}}{2}-\frac{1}{4} l_{\mu}\right)+n_{l}^{2} T_{F}^{2}\left(-\frac{209}{162}+\frac{\pi^{2}}{27}+\frac{26}{27} l_{\mu}-\frac{2}{9} l_{\mu}^{2}\right)\right]\right\},
\end{aligned}
$$

where $l_{\mu}=\ln \left(2 \mu / \mu_{s}\right), \mu$ denotes the Wilsonian cut-off and $\mu_{s}$ is the renormalization scale of the strong coupling constant. The color factors of the $\mathrm{SU}\left(N_{C}\right)$ gauge group are given by $C_{F}=\left(N_{C}^{2}-1\right) /\left(2 N_{C}\right), C_{A}=N_{C}$, and $T_{F}=1 / 2$, and the strong coupling constant is defined in the $n_{l}$ flavor theory, where $n_{l}$ denotes the number of light quark fields. Note that in our calculation no effects of finite charm quark masses are taken into account. The two-loop result of Eq. (4) and the $n_{l}^{2}$ term at three loops agree with Ref. [16].

Next, we replace the pole mass on the rhs of Eq. (4) by the $\overline{\mathrm{MS}}$ mass using results up to three loops [19,37,38]. Also here we use $\alpha_{s}^{\left(n_{l}\right)}$ as the expansion parameter. In order to obtain compact expressions, we identify the renormalization scales of the $\overline{\mathrm{MS}}$ parameters $\alpha_{s}$ and $\bar{m}$ and furthermore specify the color factors to QCD $\left(N_{C}=3\right)$. This leads to 


$$
\begin{aligned}
\frac{m^{\mathrm{kin}}}{\bar{m}}= & 1+\frac{\alpha_{s}^{\left(n_{l}\right)}}{\pi}\left(\frac{4}{3}+l_{m}-\frac{16}{9} \frac{\mu}{\bar{m}}-\frac{2}{3} \frac{\mu^{2}}{\bar{m}^{2}}\right)+\left(\frac{\alpha_{s}^{\left(n_{l}\right)}}{\pi}\right)^{2}\left\{\frac{307}{32}+\frac{\pi^{2}}{3}-\frac{\zeta_{3}}{6}+\frac{\pi^{2}}{9} l_{2}+\frac{509}{72} l_{m}+\frac{47}{24} l_{m}^{2}-n_{l}\left(\frac{71}{144}+\frac{\pi^{2}}{18}\right.\right. \\
& \left.+\frac{13}{36} l_{m}+\frac{1}{12} l_{m}^{2}\right)+\frac{\mu}{\bar{m}}\left[-\frac{860}{27}+\frac{8 \pi^{2}}{9}+\frac{88}{9} l_{\mu}+n_{l}\left(\frac{128}{81}-\frac{16}{27} l_{\mu}\right)\right]+\frac{\mu^{2}}{\bar{m}^{2}}\left[-\frac{83}{9}+\frac{\pi^{2}}{3}+\frac{2}{3} l_{m}+\frac{11}{3} l_{\mu}\right. \\
& \left.\left.+n_{l}\left(\frac{13}{27}-\frac{2}{9} l_{\mu}\right)\right]\right\}+\left(\frac{\alpha_{s}^{\left(n_{l}\right)}}{\pi}\right)^{3}\left\{\frac{8462917}{93312}+\frac{652841 \pi^{2}}{38880}+\frac{58 \zeta_{3}}{27}-\frac{695 \pi^{4}}{7776}-\frac{220 a_{4}}{27}-\frac{1439 \pi^{2} \zeta_{3}}{432}+\frac{1975 \zeta_{5}}{216}\right. \\
& -\frac{575 \pi^{2}}{162} l_{2}-\frac{22 \pi^{2}}{81} l_{2}^{2}-\frac{55}{162} l_{2}^{4}+l_{m}\left(\frac{93391}{1296}+\frac{13 \pi^{2}}{6}-\frac{23 \zeta_{3}}{12}+\frac{13 \pi^{2}}{18} l_{2}\right)+\frac{21715}{864} l_{m}^{2}+\frac{1861}{432} l_{m}^{3}+n_{l}\left[-\frac{231847}{23328}\right. \\
& -\frac{991 \pi^{2}}{648}-\frac{241 \zeta_{3}}{72}+\frac{61 \pi^{4}}{1944}+\frac{8 a_{4}}{27}-\frac{11 \pi^{2}}{81} l_{2}+\frac{2 \pi^{2}}{81} l_{2}^{2}+\frac{1}{81} l_{2}^{4}-l_{m}\left(\frac{5171}{648}+\frac{17 \pi^{2}}{36}+\frac{7 \zeta_{3}}{9}+\frac{\pi^{2}}{27} l_{2}\right)-\frac{385}{144} l_{m}^{2} \\
& \left.-\frac{43}{108} l_{m}^{3}\right]+n_{l}^{2}\left[\frac{2353}{23328}+\frac{13 \pi^{2}}{324}+\frac{7 \zeta_{3}}{54}+l_{m}\left(\frac{89}{648}+\frac{\pi^{2}}{54}\right)+\frac{13}{216} l_{m}^{2}+\frac{1}{108} l_{m}^{3}\right]+\frac{\mu}{\bar{m}}\left[-\frac{130867}{162}+\frac{1022 \pi^{2}}{27}\right. \\
& +114 \zeta_{3}-\frac{2 \pi^{4}}{3}+l_{\mu}\left(\frac{10072}{27}-\frac{88 \pi^{2}}{9}\right)-\frac{484}{9} l_{\mu}^{2}+n_{l}\left(\frac{20047}{243}-\frac{208 \pi^{2}}{81}-\frac{140 \zeta_{3}}{27}+l_{\mu}\left(-\frac{3356}{81}+\frac{16 \pi^{2}}{27}\right)\right. \\
& \left.\left.+\frac{176}{27} l_{\mu}^{2}\right)+n_{l}^{2}\left(-\frac{1292}{729}+\frac{8 \pi^{2}}{243}+\frac{256}{243} l_{\mu}-\frac{16}{81} l_{\mu}^{2}\right)\right]+\frac{\mu^{2}}{\bar{m}^{2}}\left[-\frac{22055}{108}+\frac{437 \pi^{2}}{36}+\frac{1535 \zeta_{3}}{36}-\frac{\pi^{4}}{4}+\frac{2 \pi^{2}}{27} l_{2}\right. \\
& +l_{m}\left(\frac{1409}{108}-\frac{\pi^{2}}{3}\right)+l_{\mu}\left(\frac{689}{6}-\frac{11 \pi^{2}}{3}\right)-\frac{11}{3} l_{m} l_{\mu}+\frac{23}{36} l_{m}^{2}-\frac{121}{6} l_{\mu}^{2}+n_{l}\left(\frac{1699}{81}-\frac{8 \pi^{2}}{9}-\frac{35 \zeta_{3}}{18}-\frac{13}{18} l_{m}\right. \\
& \left.\left.\left.+l_{\mu}\left(-\frac{691}{54}+\frac{2 \pi^{2}}{9}\right)+\frac{2}{9} l_{m} l_{\mu}-\frac{1}{18} l_{m}^{2}+\frac{22}{9} l_{\mu}^{2}\right)+n_{l}^{2}\left(-\frac{209}{486}+\frac{\pi^{2}}{81}+\frac{26}{81} l_{\mu}-\frac{2}{27} l_{\mu}^{2}\right)\right]\right\},
\end{aligned}
$$

with $\bar{m}=\bar{m}\left(\mu_{s}\right)$ and

$$
l_{m}=\ln \frac{\mu_{s}^{2}}{\bar{m}^{2}}, \quad l_{2}=\ln 2, a_{4}=\mathrm{Li}_{4}\left(\frac{1}{2}\right) .
$$

We are now in the position to specify our results to the charm and bottom quark systems and check the perturbative stability of the quark mass relations.

The input values for our numerical analysis are $\alpha_{s}^{(5)}\left(M_{Z}\right)=0.1179$ [39], $\bar{m}_{c}(3 \mathrm{GeV})=0.993 \mathrm{GeV}$ [40] and $\bar{m}_{b}\left(\bar{m}_{b}\right)=4.163 \mathrm{GeV}$ [41]. We use RUNDEC [42] for the running of the $\overline{\mathrm{MS}}$ parameters and the decoupling of heavy particles. For the Wilsonian cut-off we choose $\mu=1 \mathrm{GeV}$ for bottom [6] and $\mu=0.5 \mathrm{GeV}$ for charm [43].

Let us start with the charm quark where we have $n_{l}=3$. We aim for a relation between $m_{c}^{\text {kin }}$ and $\bar{m}_{c}\left(\mu_{s}\right)$ for different choices of $\mu_{s}$. Often numerical values for $\bar{m}_{c}\left(\bar{m}_{c}\right)$ are provided. However, this choice suffers from small renormalization scales of the order $1 \mathrm{GeV}$. A more appropriate choice is thus $\bar{m}_{c}(2 \mathrm{GeV})$ or $\bar{m}_{c}(3 \mathrm{GeV})$. For the three choices we obtain the following perturbative expansions

$$
\begin{aligned}
& m_{c}^{\mathrm{kin}}=993+191+100+52 \mathrm{MeV}=1336 \mathrm{MeV}, \\
& m_{c}^{\mathrm{kin}}=1099+163+76+34 \mathrm{MeV}=1372 \mathrm{MeV}, \\
& m_{c}^{\text {kin }}=1279+84+30+11 \mathrm{MeV}=1404 \mathrm{MeV},
\end{aligned}
$$

where from top to bottom $\mu_{s}=3 \mathrm{GeV}, 2 \mathrm{GeV}$, and $\bar{m}_{c}$ have been chosen. Within each equation the four numbers after the first equality sign refer to the tree-level results and the one-, two-, and three-loop corrections. One observes that for each choice of $\mu_{s}$ the perturbative expansion behaves reasonably. The three-loop terms range from $11 \mathrm{MeV}$ to $52 \mathrm{MeV}$ and roughly cover the splitting of the final numbers for $m_{c}^{\mathrm{kin}}(0.5 \mathrm{GeV})$.

In the case of the bottom quark, we follow Ref. [21] and adapt two different schemes for the charm quark: we either consider the charm quark as decoupled and set $n_{l}=3$, or we set $n_{l}=4$, which corresponds to $m_{c}=0$. (In the latter case one could include $m_{c} / m_{b}$ corrections, which we postpone to a future analysis [22].)

Using $\bar{m}_{b}\left(\bar{m}_{b}\right)$ as input we obtain the following results for the kinetic mass

$$
\begin{aligned}
& m_{b}^{\text {kin }}=4163+248+81+30 \mathrm{MeV}=4521 \mathrm{MeV}, \\
& m_{b}^{\text {kin }}=4163+259+77+25 \mathrm{MeV}=4523 \mathrm{MeV},
\end{aligned}
$$


where the top and bottom line correspond to $n_{l}=3$ and $n_{l}=4$, respectively. In both cases we observe a good convergence of the perturbative series: the coefficients reduce by factors between $\approx 2.5$ and $\approx 3.5$ when including higher orders. We estimate the unknown four-loop corrections and contributions from higher dimensional operators, which scale as $\alpha_{s} \mu^{3} / m_{b}^{3} \sim \alpha_{s}^{4}$, by $50 \%$ of the three-loop corrections and assign an uncertainty of $15 \mathrm{MeV}$ and $12 \mathrm{MeV}$ for $n_{l}=3$ and $n_{l}=4$, respectively. Note that our $\bar{m}_{b}-m_{b}^{\text {kin }}$ scheme-conversion uncertainties are now smaller than the error of $m_{b}^{\mathrm{kin}}$ as determined by global fits: $m_{b}^{\text {kin }}(1 \mathrm{GeV})=4554 \pm 18 \mathrm{MeV}[1]$.

For the computation of $\bar{m}_{b}\left(\bar{m}_{b}\right)$ from the kinetic mass, we proceed as follows. We first use the inverted version of Eq. (5) to compute the $\overline{\mathrm{MS}}$ bottom quark mass at the scale $\mu_{s}=m_{b}^{\text {kin }}$. Afterwards, we use the QCD renormalization group equations at five-loop accuracy [44-50] as implemented in RUNDEC [42] to run to $\mu_{s}=\bar{m}_{b}$. In order to demonstrate the perturbative series, we choose $m_{b}^{\mathrm{kin}}$ from Eq. (8) and obtain for $n_{l}=3$ and $n_{l}=4$

$$
\begin{aligned}
& \bar{m}_{b}\left(m_{b}^{\text {kin }}\right)=4521-273-101-39 \mathrm{MeV}, \\
& \bar{m}_{b}\left(m_{b}^{\text {kin }}\right)=4523-286-98-34 \mathrm{MeV},
\end{aligned}
$$

with similar convergence properties as in Eq. (8). Thus, we estimate the uncertainty from unknown higher order corrections as $\pm 18 \mathrm{MeV}$ and $\pm 17 \mathrm{MeV}$, respectively. In an alternative approach, one can estimate the uncertainty from the variation of the intermediate scale $\mu_{s}$ which leads to similar uncertainty estimates.

Finally, we present simple formulas that can be used to convert the scale-invariant bottom quark mass to the kinetic scheme or vice versa using the preferred input values for the mass and strong coupling constant. We have

$$
\begin{aligned}
\frac{\bar{m}_{b}\left(\bar{m}_{b}\right)}{\mathrm{MeV}} & =4163+\Delta_{\text {kin }}^{\left(n_{l}\right)}\{13,13\}-\Delta_{\alpha_{s}}\{7,7\} \pm\{18,17\}, \\
\frac{m_{b}^{\text {kin }}}{\mathrm{MeV}} & =4522+\Delta_{\overline{\mathrm{MS}}}\{18,18\}+\Delta_{\alpha_{s}}\{8,8\} \pm\{15,12\},
\end{aligned}
$$

where the first (second) number in the curly brackets corresponds to $n_{l}=3\left(n_{l}=4\right)$. Furthermore, we have defined $\Delta_{\mathrm{kin}}^{(3)}=\left(m_{b}^{\mathrm{kin}} / \mathrm{MeV}-4518\right) / 15, \Delta_{\mathrm{kin}}^{(4)}=\left(m_{b}^{\mathrm{kin}} / \mathrm{MeV}-4520\right) / 15$, $\Delta_{\overline{\mathrm{MS}}}=\left(\bar{m}_{b}\left(\bar{m}_{b}\right) / \mathrm{MeV}-4163\right) / 16$, and $\Delta_{\alpha_{s}}=\left(\alpha_{s}-0.1179\right) /$ 0.001 .

Conclusions.-The main purpose of this Letter is the improvement of the precision in the conversion relation between the heavy quark kinetic and $\overline{\mathrm{MS}}$ masses. This goal is reached by computing the relation between the kinetic and pole mass to three-loop order; previously only twoloop corrections, supplemented by large- $\beta_{0}$ terms, were available. The main results of this paper can be found in
Eqs. (4) and (5). Using a conservative uncertainty estimate, the new corrections reduce the uncertainty in transformation formulas by about a factor of 2. Our findings constitute important ingredients in the extraction of $\left|V_{c b}\right|$ at the percent level or even below.

We thank Andrzej Czarnecki and Mikołaj Misiak for useful discussions and communications and Alexander Smirnov for help in the use of ASY [27]. We also thank Joshua Davies and Thomas Mannel for carefully reading the manuscript. We are grateful to Florian Herren for providing us his program that automates the partial fraction decomposition in case of linearly dependent denominators. This research was supported by the Deutsche Forschungsgemeinschaft (DFG, German Research Foundation) under Grant No. 396021762-TRR 257 "Particle Physics Phenomenology after the Higgs Discovery."

*Corresponding author. matteo.fael@kit.edu Corresponding author. kay.schoenwald@kit.edu Corresponding author. matthias.steinhauser@kit.edu

[1] Y.S. Amhis et al. (HFLAV Collaboration), arXiv: 1909.12524

[2] A. J. Buras and R. Fleischer, Quark Mixing, CP Violation and Rare Decays After the Top Quark Discovery, Advanced Series on Directions in High Energy Physics Vol. 15 (World Scientific, Singapore, 1998), pp. 65-238.

[3] J. Brod, M. Gorbahn, and E. Stamou, Phys. Rev. D 83, 034030 (2011).

[4] C. Bobeth, M. Gorbahn, T. Hermann, M. Misiak, E. Stamou, and M. Steinhauser, Phys. Rev. Lett. 112, 101801 (2014).

[5] Z. Ligeti and F. Sala, J. High Energy Phys. 09 (2016) 083.

[6] P. Gambino and C. Schwanda, Phys. Rev. D 89, 014022 (2014).

[7] A. Alberti, P. Gambino, K. J. Healey, and S. Nandi, Phys. Rev. Lett. 114, 061802 (2015).

[8] P. Gambino, K. J. Healey, and S. Turczyk, Phys. Lett. B 763, 60 (2016).

[9] I. I. Bigi, M. A. Shifman, N. Uraltsev, and A. I. Vainshtein, Phys. Rev. D 56, 4017 (1997).

[10] A. Hoang and T. Teubner, Phys. Rev. D 60, 114027 (1999).

[11] A. H. Hoang, Z. Ligeti, and A. V. Manohar, Phys. Rev. D 59, 074017 (1999).

[12] A. H. Hoang, Z. Ligeti, and A. V. Manohar, Phys. Rev. Lett. 82, 277 (1999).

[13] C. W. Bauer, Z. Ligeti, M. Luke, A. V. Manohar, and M. Trott, Phys. Rev. D 70, 094017 (2004).

[14] P. Marquard, A. V. Smirnov, V. A. Smirnov, and M. Steinhauser, Phys. Rev. Lett. 114, 142002 (2015).

[15] P. Marquard, A. V. Smirnov, V. A. Smirnov, M. Steinhauser, and D. Wellmann, Phys. Rev. D 94, 074025 (2016).

[16] A. Czarnecki, K. Melnikov, and N. Uraltsev, Phys. Rev. Lett. 80, 3189 (1998). 
[17] M. Beneke and V. M. Braun, Nucl. Phys. B426, 301 (1994).

[18] I. I. Y. Bigi, M. A. Shifman, N. G. Uraltsev, and A. I. Vainshtein, Phys. Rev. D 50, 2234 (1994).

[19] K. Melnikov and T. v. Ritbergen, Phys. Lett. B 482, 99 (2000).

[20] I. I. Y. Bigi, M. A. Shifman, N. G. Uraltsev, and A. I. Vainshtein, Phys. Rev. D 52, 196 (1995).

[21] P. Gambino, J. High Energy Phys. 09 (2011) 055.

[22] M. Fael, K. Schönwald, and M. Steinhauser (to be published).

[23] M. Beneke and V. A. Smirnov, Nucl. Phys. B522, 321 (1998).

[24] V. A. Smirnov, Springer Tracts Mod. Phys. 250, 1 (2012).

[25] P. Nogueira, J. Comput. Phys. 105, 279 (1993).

[26] B. Ruijl, T. Ueda, and J. Vermaseren, arXiv:1707.06453.

[27] A. Pak and A. Smirnov, Eur. Phys. J. C 71, 1626 (2011).

[28] A. V. Smirnov and F. S. Chuharev, Comput. Phys. Commun. 247, 106877 (2020).

[29] R. N. Lee, arXiv:1212.2685; J. Phys. Conf. Ser. 523, 012059 (2014).

[30] C. Schneider, Sém. Lothar. Combin. 56, 1 (2007), article B56b; in Computer Algebra in Quantum Field Theory: Integration, Summation and Special Functions, edited by C. Schneider and J. Blümlein, Texts and Monographs in Symbolic Computation (Springer, Wien, 2013), p. 325.

[31] J. Ablinger, J. Blümlein, S. Klein, and C. Schneider, Nucl. Phys. B, Proc. Suppl. 205-206, 110 (2010); J. Blümlein, A. Hasselhuhn, and C. Schneider, Proc. Sci., RADCOR2011 (2011) 032 [arXiv:1202.4303]; C. Schneider, J. Phys. Conf. Ser. 523, 012037 (2014).

[32] J. Vermaseren, Int. J. Mod. Phys. A 14, 2037 (1999); E. Remiddi and J. Vermaseren, Int. J. Mod. Phys. A 15, 725 (2000); J. Blümlein, Comput. Phys. Commun. 180, 2218 (2009); J. Ablinger, Diploma thesis, J. Kepler University Linz, 2009; J. Ablinger, J. Blümlein, and C. Schneider, J. Math. Phys. (N.Y.) 52, 102301 (2011); J. Math. Phys. (N.Y.) 54, 082301 (2013); J. Ablinger, Ph. D. thesis, J. Kepler University Linz, 2012; J. Ablinger, J. Blümlein, and C. Schneider, J. Phys. Conf. Ser. 523, 012060 (2014); J. Ablinger, J. Blümlein, C. Raab, and C. Schneider, J. Math. Phys. (N.Y.) 55, 112301 (2014); J. Ablinger, Proc. Sci.,
LL2014 (2014) 019 [arXiv:1407.6180]; arXiv:1606.02845; Proc. Sci., RADCOR2017 (2017) 069 [arXiv:1801.01039]; Proc. Sci., LL2018 (2018) 063; arXiv:1902.11001.

[33] H. R. P. Ferguson and D. H. Bailey, RNR Technical Report No. RNR-91-032, 1992; H. R. P. Ferguson, D. H. Bailey, and S. Arno, NASA Technical Report No. NAS-96-005, 1996.

[34] A. Kotikov, Phys. Lett. B 254, 158 (1991).

[35] T. Gehrmann and E. Remiddi, Nucl. Phys. B580, 485 (2000).

[36] J. M. Henn, Phys. Rev. Lett. 110, 251601 (2013).

[37] K. G. Chetyrkin and M. Steinhauser, Phys. Rev. Lett. 83, 4001 (1999).

[38] K. G. Chetyrkin and M. Steinhauser, Nucl. Phys. B573, 617 (2000).

[39] M. Tanabashi et al. (Particle Data Group), Phys. Rev. D 98, 030001 (2018).

[40] K. G. Chetyrkin, J. H. Kühn, A. Maier, P. Maierhofer, P. Marquard, M. Steinhauser, and C. Sturm, Phys. Rev. D 96, 116007 (2017).

[41] K. G Chetyrkin, J. H. Kühn, A. Maier, P. Maierhofer, P. Marquard, M. Steinhauser, and C. Sturm, Phys. Rev. D 80, 074010 (2009).

[42] F. Herren and M. Steinhauser, Comput. Phys. Commun. 224, 333 (2018).

[43] P. Gambino and J. F. Kamenik, Nucl. Phys. B840, 424 (2010).

[44] P. Baikov, K. Chetyrkin, and J. Kühn, J. High Energy Phys. 10 (2014) 076.

[45] T. Luthe, A. Maier, P. Marquard, and Y. Schroder, J. High Energy Phys. 01 (2017) 081.

[46] P. Baikov, K. Chetyrkin, and J. Kühn, J. High Energy Phys. 04 (2017) 119.

[47] P. A. Baikov, K. G. Chetyrkin, and J. H. Kühn, Phys. Rev. Lett. 118, 082002 (2017).

[48] F. Herzog, B. Ruijl, T. Ueda, J. A. M. Vermaseren., and A. Vogt, J. High Energy Phys. 02 (2017) 090.

[49] T. Luthe, A. Maier, P. Marquard, and Y. Schroder, J. High Energy Phys. 10 (2017) 166.

[50] K. Chetyrkin, G. Falcioni, F. Herzog, and J. Vermaseren, J. High Energy Phys. 10 (2017) 179. 